\title{
THE POTENTIAL FUNCTION METHOD FOR THE SOLUTION OF TWO-DIMENSIONAL STRESS PROBLEMS*
}

\author{
BY \\ C. W. MACGREGOR \\ I. INTRODUCTION
}

At different intervals during the development of the theory of elasticity various methods of solution for two-dimensional stress problems have been proposed. Among these may be mentioned methods based on the use of the Airy stress function $†$; the strain energy function $f$; the recently developed socalled "displacement function" $\$$; and the potential function.\| The latter method was originally suggested independently by S. D. Carothers $\|$ and by A. Nâdai $\|$; and although a useful and convenient one it does not as yet seem to have found broader application.

In his derivation, S. D. Carothers obtained the expressions for the stress components from solutions of the stress equations of equilibrium and the identical relations between strain components, while A. Nádai derived them for two important special cases by a considerably shorter method which will be developed further in the present investigation. More recently, the potential method has also been discussed and applied to various problems by L.Föppl ${ }^{\top}$, E. Kohl, ${ }^{* *}$ and H. Neuber. †† Both Föppl and Kohl derived the general expressions for the stress components from solutions of the fundamental

\footnotetext{
* Presented to the Society, April 20, 1935; received by the editors November 1, 1934.

$\dagger$ For details cf. Love, A. E. H., Mathematical Theory of Elasticity, London, Cambridge University Press, 4th edition, 1927, p. $88 \mathrm{ff}$.

Timoshenko, S., Theory of Elasticity, New York, McGraw-Hill, 1st edition, 1934, p. 25 ff.

$\ddagger$ Timoshenko, S., The approximate solution of two dimensional problems in elasticity, Philosophical Magazine, vol. 47 (1924), pp. 1095-1104.

§ Marguerre, K., Spannungsverteilung und Wellenausbreitung in der kontinuierlich gestützten Platte, Ingenieur-Archiv, vol. 4 (1933), pp. 332-353.

|| Nádai, A., Darstellung ebener Spannungszustände mit Hilfe von winkeltreuen Abbildungen, Zeitschrift für Physik, vol. 41 (1927), pp. 49-50.

Carothers, S. D., The direct determination of stress, Proceedings of the Royal Society of London, vol. 97 (1920), p. $110 \mathrm{ff}$.

T Föppl, L., Konforme Abbildungen ebener Spannungszustände, Zeitschrift für Angewandte Mathematik und Mechanik, vol. 11 (1931), pp. 81-92.

** Kohl, E., Beitrag zur Lösung des ebenen Spannungsproblems, Zeitschrift für Angewandte Mathematik und Mechanik, vol. 10 (1930), p. 141.

†† Neuber, H., Elastisch-strenge Lösungen zur Kerbwirkung bei Scheiben und Umdrehungskörpern, Zeitschrift für Angewandte Mathematik und Mechanik, vol. 13 (1933), pp. 439-443.
} 
elastic equations in terms of displacements. It will be shown later that these expressions for the general stress components may also be derived by following a somewhat different procedure.

It is the intention here in particular to deal with the potential method more completely than has been done heretofore and in a somewhat different manner, and to apply it to a number of examples.

\section{Notation}

$p_{0}, \tau_{1}$ : distributed normal and shearing forces per unit area. $\sigma_{x}, \sigma_{y}, \tau_{x y}$ : normal and shearing stresses in the $[x, y]$ plane.

$\boldsymbol{\epsilon}_{x}, \boldsymbol{\epsilon}_{y}, \gamma_{x y}$ : strain components in the $[x, y]$ plane.

$\xi, \eta$ : displacements in the $x$ and $y$ directions.

$E, G, \nu$ : moduli of elasticity and rigidity, Poisson's ratio.

$F$ : Airy's stress function.

$c$ : a unit of distance.

$z, \bar{z}$ : complex variables $x+i y$ and $x-i y$ respectively. $\Phi, \Psi, \chi, \psi:$ potential functions where $W(z)=\Psi-i \Phi, K(z)=\chi+i \psi$.

Re: real part of.

$Z(z), H(z)$ : functions of a complex variable where $Z(z)=\Theta+i \Omega$ and $H(z)$ $=\Theta_{0}+i \Omega_{0}$.

II. GENERAL EXPRESSIONS FOR STRESSES IN TERMS OF POTENTIAL FUNCTIONS OR FUNCTIONS OF A COMPLEX VARIABLE

Following Airy's stress function method for the solution of stress problems in the plane, the stresses will be completely determined if a function $F[x, y]$ can be found which is a solution of the biharmonic equation

$$
\nabla^{2} \nabla^{2} F=0 \text {, }
$$

where $\nabla^{2}$ represents the Laplacean operator in two dimensions, and which when substituted in the expressions for the stresses

$$
\sigma_{x}=\frac{\partial^{2} F}{\partial y^{2}}, \quad \sigma_{y}=\frac{\partial^{2} F}{\partial x^{2}}, \quad \tau_{x y}=-\frac{\partial^{2} F}{\partial x \partial y}
$$

satisfies the boundary conditions of the problem. The general solution of equation (1) may be expressed in any one of the following equivalent forms*:

$$
F=y \Theta+\Theta_{0}=x \Theta_{2}+\Theta_{1}=\left(x^{2}+y^{2}\right) \Theta_{4}+\Theta_{3},
$$

where the functions $\Theta_{i}$ are various logarithmic potential functions. Hence any

* Cf. Selected Problems in the Theories of Flat Plates and Plane Stress, Dissertation, University of Pittsburgh, 1934. 
one of these forms may be used to express the most general biharmonic stress function in two dimensions. Selecting the first form of equations (3) or $y \Theta+\Theta_{0}$ and substituting in equations (2) making use of the relations

$\Phi=\frac{\partial \Theta}{\partial y}, \quad \Psi=\frac{\partial \Theta}{\partial x}, \quad \chi=-\frac{\partial \Theta_{0}}{\partial x}, \quad \psi=\frac{\partial \Theta_{0}}{\partial y}, \quad W(z)=\Psi-i \Phi$,

we obtain

$$
\begin{aligned}
\sigma_{x} & =2 \Phi+y \frac{\partial \Phi}{\partial y}+\frac{\partial \chi}{\partial x} \\
\sigma_{y} & =-y \frac{\partial \Phi}{\partial y}-\frac{\partial \chi}{\partial x} \\
\tau_{x y} & =-\Psi-y \frac{\partial \Phi}{\partial x}+\frac{\partial \chi}{\partial y}
\end{aligned}
$$

which are the general expressions for the stresses in two dimensions in terms of logarithmic potential functions only. These equations hold for a body of any shape stressed in its plane. Considering the special case of the semi-plane, equations (4) may be put in a simpler form treating the cases of normal and shear loading separately. For normal loading only along the line $y=0$ of the semi-plane, it follows that $\Psi=\partial \chi / \partial y$ and from the Cauchy-Riemann equations we find that $\Phi=-\partial \chi / \partial x$. Hence for this case equations (4) become

$$
\begin{gathered}
\sigma_{x}=\Phi+y \frac{\partial \Phi}{\partial y}, \quad \sigma_{y}=\Phi-y \frac{\partial \Phi}{\partial y}, \\
\tau_{x y}=-y \frac{\partial \Phi}{\partial x}
\end{gathered}
$$

which show that along $y=0, \sigma_{y}=\Phi, \tau_{x y}=0$. In a similar manner for shear loading only along the line $y=0$ of the semi-plane, placing $\chi=0$ equations (4) reduce to

(6)

$$
\begin{aligned}
\sigma_{x}= & 2 \Phi+y \frac{\partial \Phi}{\partial y} \\
\sigma_{y}= & -y \frac{\partial \Phi}{\partial y} \\
\tau_{x y}= & -\Psi-y \frac{\partial \Phi}{\partial x},
\end{aligned}
$$

indicating that for $y=0, \sigma_{y}=0, \tau_{x y}=-\Psi$. The stress problem for these two cases has thus been reduced to the first boundary value problem of the potential theory. By computing the dilatation and rotation for the stresses given 
in equations (5) and (6) it can be seen that the physical meaning of the potential functions $\Phi(x, y)$ and $\Psi(x, y)$ is that they represent to a constant factor the dilatation and rotation respectively.

There are several inherent advantages in having the stress components given in terms of functions of a complex variable and its derivatives. The advantages are (a) less labor involved in the computation of a given case; (b) it being unnecessary to compute the conjugate function which in most cases is tedious and often difficult; and (c) greater ease in recognizing the necessary function $W(z)$ for a given load distribution on the semi-plane than its real or imaginary parts.

The stress components in complex form may be derived either by making use of the stress equations already obtained, and of certain relations between the potential functions, or from the beginning in complex form. The latter method will be followed here. Following a suggestion made by Busemann* we may express the biharmonic stress function as

$$
F(x, y)=F\left(\frac{z+\bar{z}}{2}, \frac{z-\bar{z}}{2 i}\right)=F_{1}(z, \bar{z})
$$

where $F_{1}$ is real. Substituting this stress function in equations (2) and differentiating, using the relations

$$
\frac{\partial}{\partial x}=\frac{\partial}{\partial z}+\frac{\partial}{\partial \bar{z}}, \quad \frac{\partial}{\partial y}=i\left(\frac{\partial}{\partial z}-\frac{\partial}{\partial \bar{z}}\right)
$$

we get, if

$$
F(x, y)=F_{1}(z, \bar{z})=\operatorname{Re}\left(H(z)+\frac{i(\bar{z}-z)}{2} Z(z)\right)
$$

where

$$
H(z)=\Theta_{0}+i \Omega_{0}, \quad Z(z)=\Theta+i \Omega,
$$

and

$$
\begin{aligned}
& \frac{d H(z)}{d z}=-K(z)=-\chi-i \psi, \\
& \frac{d Z(z)}{d z}=W(z)=\Psi-i \Phi,
\end{aligned}
$$

the general expressions for the stress components in complex form as

* Busemann, A., Schematischer Übergang von Vectorgleichungen auf komplexe Gleichungen bei ebenen Problemen, Zeitschrift für Angewandte Mathematik und Mechanik, vol. 11 (1931), pp. 71-72. 


$$
\begin{aligned}
\sigma_{x} & =\operatorname{Re}\left[2 i W(z)-y \frac{d W(z)}{d z}+\frac{d K(z)}{d z}\right] \\
\sigma_{y} & =\operatorname{Re}\left[y \frac{d W(z)}{d z}-\frac{d K(z)}{d z}\right] \\
\tau_{x y} & =\operatorname{Re}\left[-W(z)-i y \frac{d W(z)}{d z}+i \frac{d K(z)}{d z}\right]
\end{aligned}
$$

Using certain obvious relations between the complex functions, we get for the special case of normal loading on the semi-plane

$$
\begin{aligned}
\sigma_{x} & =\operatorname{Re}\left[i W(z)-y \frac{d W(z)}{d z}\right], \\
\sigma_{y} & =\operatorname{Re}\left[i W(z)+y \frac{d W(z)}{d z}\right], \\
\tau_{x y} & =\operatorname{Re}\left[-i y \frac{d W(z)}{d z}\right],
\end{aligned}
$$

and for the special case of shear loading on the semi-plane,

$$
\begin{aligned}
\sigma_{x} & =\operatorname{Re}\left[2 i W(z)-y \frac{d W(z)}{d z}\right], \\
\sigma_{y} & =\operatorname{Re}\left[y \frac{d W(z)}{d z}\right], \\
\tau_{x y} & =\operatorname{Re}\left[-W(z)-i y \frac{d W(z)}{d z}\right] .
\end{aligned}
$$

In equations (10) and (11), the boundary $y=0$ is loaded by normal pressure $\sigma_{y}=\operatorname{Re}[i W(z)]_{y=0}$ and shear pressure $\tau_{x y}=\operatorname{Re}[-W(z)]_{y=0}$ respectively.

\section{GENERAL EXPRESSIONS FOR DISPLACEMENTS IN}

\section{TERMS OF POTENTIAL FUNCTIONS}

The expressions for the displacements corresponding to the states of stress described in the preceding section may now easily be calculated. Consider a function $Z(z)=\Theta+i \Omega$ which is analogous to the so-called complex stream function of hydrodynamics. Then

$$
\frac{d Z(z)}{d z}=\frac{\partial \Theta}{\partial x}+i \frac{\partial \Omega}{\partial x}=\frac{\partial \Theta}{\partial x}-i \frac{\partial \Theta}{\partial y}=\Psi-i \Phi=W(z)
$$

which follows from relations used in the previous section. In the latter equa- 
tions $W(z)$ is analogous to the complex velocity function in fluid dynamics. Hence it follows that

$$
\Phi=\frac{\partial \Theta}{\partial y}=-\frac{\partial \Omega}{\partial x} ; \quad \Psi=\frac{\partial \Theta}{\partial x}=\frac{\partial \Omega}{\partial y} .
$$

The general expressions for the stress components given in equations (4) may now be substituted in

$$
\begin{aligned}
\epsilon_{x} & =\frac{\partial \xi}{\partial x}=\frac{1}{2 G}\left[\sigma_{x}(1-\nu)-\nu \sigma_{y}\right], \\
\epsilon_{y} & =\frac{\partial \eta}{\partial y}=\frac{1}{2 G}\left[\sigma_{y}(1-\nu)-\nu \sigma_{x}\right], \\
\gamma_{x y} & =\frac{\partial \xi}{\partial y}+\frac{\partial \eta}{\partial x}=\frac{\tau_{x y}}{G},
\end{aligned}
$$

which hold for plane strain $\left(\epsilon_{z}=0\right)$. If the resulting equations are then integrated for the displacements $(\xi, \eta)$ making use of (12), the general expressions for the displacements in plane strain become

$$
\begin{aligned}
& \xi=\frac{1}{2 G}\left[-2(1-v) \Omega-y \frac{\partial \Omega}{\partial y}+\chi\right]+c_{1} y+c_{2}, \\
& \eta=\frac{1}{2 G}\left[(1-2 v) \Theta-y \frac{\partial \Theta}{\partial y}-\psi\right]-c_{1} x+c_{3} .
\end{aligned}
$$

By introducing the relations between the potential functions and the biharmonic stress function, and through further relations between the potential functions themselves, equations (14) may be put in the forms

$$
\begin{aligned}
& \xi=\frac{1}{2 G}\left[-2(1-\nu) \Omega-\frac{\partial F}{\partial x}\right]+c_{1} y+c_{2}, \\
& \eta=\frac{1}{2 G}\left[2(1-\nu) \Theta-\frac{\partial F}{\partial y}\right]-c_{1} x+c_{3},
\end{aligned}
$$

which hold for the general case of plane strain. The general displacement components in plane stress may then be obtained from equations (15) merely by replacing $(1-\nu)$ by $1 /(1+\nu)$.

In a similar manner, the displacements in plane strain may be obtained for the special cases of normal and shear loadings on the boundary of the semi-plane. These become 


$$
\begin{aligned}
& \xi=\frac{1}{2 G}\left[-(1-2 \nu) \Omega-y \frac{\partial \Omega}{\partial y}\right]-c_{1} y+c_{3}, \\
& \eta=\frac{1}{2 G}\left[2(1-\nu) \Theta-y \frac{\partial \Theta}{\partial y}\right]+c_{1} x+c_{2}
\end{aligned}
$$

for the case of normal pressure, and

$$
\begin{aligned}
& \xi=\frac{1}{2 G}\left[-2(1-\nu) \Omega-y \frac{\partial \Omega}{\partial y}\right]-c_{1} y+c_{3}, \\
& \eta=\frac{1}{2 G}\left[(1-2 \nu) \Theta-y \frac{\partial \Theta}{\partial y}\right]+c_{1} x+c_{2}
\end{aligned}
$$

for the case of shear loading on the straight boundary of the semi-plane.

IV. Application of the METHOD to SPECIAL PROBLEMS IN tHE SEMi-PLANE

The expressions for the stress components derived in complex form are very useful in the solution of a large group of problems. In the case of the semi-plane, for example, the complex function $W(z)$ can be selected for many pressure distributions immediately from the form of the given loading. It can be shown in the case of a large number of rational or transcendental functions of the complex variable $z$ that the

$$
\operatorname{Re}[W(z)]_{y=0}=\left.\Psi(x, y)\right|_{y=0}=W(x) .
$$

Hence, for various load distributions $W(x)$ applied along the straight boundary, it is only necessary to replace $x$ by $z$ in $W(x)$ in order to obtain the complex function necessary to derive the corresponding stresses.

A particular group of problems in the semi-plane are of considerable practical importance; namely those in which only one half of the straight boundary is loaded by either normal or shear forces. For many such distributions $f(x)$ of either shear or normal pressure which can be expressed by certain rational or transcendental functions of $x$, it will be found that considerable use may be made of the function $\log (z / c)$ where $c$ is a real constant. In such cases it will be found that the corresponding complex function $W(z)$ from which the stresses are derived will be represented by

$$
W(z)=f(z) \cdot \log \frac{z}{c} .
$$

The function $\log (z / c)$ in this case provides that the pressure will be applied along one half of the boundary only. Equation (18) is quite general for functions restricted to the type mentioned, and with it various problems may be 
solved for different functions $f(z)$. In some of the examples to be discussed in this section, a simple type of function will be chosen, namely a function proportional to $z^{n}$. It is shown that with this function a considerable number of problems may be solved.

It will however be mentioned here that one should perhaps distinguish between two types of loading along the semi-plane boundary; namely those cases in which the external forces are applied along a small finite strip of the boundary or tend to zero as $r$ increases, and those cases in which the applied forces increase uniformly from the origin along $y=0$. In the former, convergence of the loads towards zero makes possible the requirement that all the stresses converge toward zero values as the distances from the origin become large. In the other case this requirement is not possible.

A. Normal pressure varying as $r^{n}$. The expressions for the stresses will be derived from two different complex functions $W(z)$ depending on whether $n$ is integral or fractional. For $n$ a positive or a negative integer, let

where

$$
W(z)=\frac{p_{0}}{\pi} i^{2 n} \frac{z^{n}}{a^{n}} \log \frac{z}{c},
$$

$$
f(z)=\frac{p_{0}}{\pi} i^{2 n} \frac{z^{n}}{a^{n}}
$$

in equation (18). Substituting this expression for $W(z)$ in equations (10) we get

$$
\begin{aligned}
\sigma_{x} & =\frac{p_{0}}{\pi a^{n}} \operatorname{Re}\left[i^{2 n}\left(i z^{n} \log \frac{z}{c}-y z^{n-1}\left[1+n \log \frac{z}{c}\right]\right)\right], \\
\sigma_{y} & =\frac{p_{0}}{\pi a^{n}} \operatorname{Re}\left[i^{2 n}\left(i z^{n} \log \frac{z}{c}+y z^{n-1}\left[1+n \log \frac{z}{c}\right]\right)\right], \\
\tau_{x y} & =-\frac{p_{0}}{\pi a^{n}} \operatorname{Re}\left[i^{2 n+1} y z^{n-1}\left(1+n \log \frac{z}{c}\right)\right],
\end{aligned}
$$

which are the general stress components for the values of $n$ mentioned above. In equations (19) are included such special cases as uniform pressure $(n=0)$, linearly increasing pressure $(n=1)$, parabolic pressure $(n=2)$, hyperbolic pressure $(n=-1)$, and various others, the explicit expressions for which may be easily obtained by replacing $n$ in equations (19) by its appropriate value.*

For $n$ a positive or a negative fraction, let

$$
W(z)=\frac{p_{0}}{\sin n \pi} \frac{z^{n}}{a^{n}},
$$

\footnotetext{
* For these explicit expressions in the special cases cf. footnote on p. 178.
} 
and by substituting these expressions in equations (10) we get after some reduction

$$
\begin{aligned}
\sigma_{x} & =\frac{p_{0}}{a^{n} \sin n \pi} \operatorname{Re}\left[r^{n}(\cos (n-1) \phi+i \sin (n-1) \phi)(i \cos \phi-(1+n) \sin \phi)\right], \\
\sigma_{y} & =\frac{p_{0}}{a^{n} \sin n \pi} \operatorname{Re}\left[r^{n}(\cos (n-1) \phi+i \sin (n-1) \phi)(i \cos \phi-(1-n) \sin \phi)\right], \\
\tau_{x y} & =\frac{p_{0}}{a^{n} \sin n \pi} \operatorname{Re}\left[r^{n}(\cos (n-1) \phi+i \sin (n-1) \phi)(-i n \sin \phi)\right],
\end{aligned}
$$

which are the general stress components for normal loading on one side of the semi-plane boundary with $n$ restricted to fractional values. In equations (20) are included such special cases as parabolic pressure $(n=1 / 2)$, hyperbolic pressure $(n=-1 / 2)$, hyperbolic pressure where $n=-5 / 16$, and many others. The explicit expressions for the stresses in these cases may also be obtained in the same manner as discussed previously.

B. Shearing forces varying as $r^{n}$. For the cases of shearing forces which vary in the same manner as the normal pressures treated in the last section, the stress components will be derived from two different complex functions $W(z)$ depending on whether $n$ is integral or fractional as before.

In case $n$ is a positive or a negative integer, consider the complex function

where

$$
W(z)=\frac{\tau_{1}}{\pi} i \frac{z^{n}}{(-a)^{n}} \log \frac{z}{c}
$$

$$
f(z)=\frac{\tau_{1}}{\pi} i \frac{z^{n}}{(-a)^{n}}
$$

in equation (18). Substituting this value of $W(z)$ in equations (11) we obtain

$$
\begin{aligned}
\sigma_{x} & =\frac{\tau_{1}}{\pi(-a)^{n}} \operatorname{Re}\left[z^{n-1}\left([-2 z-i y n] \log \frac{z}{c}-i y\right)\right], \\
\sigma_{y} & =\frac{\tau_{1}}{\pi(-a)^{n}} \operatorname{Re}\left[i y z^{n-1}\left(1+n \log \frac{z}{c}\right)\right], \\
\tau_{x y} & =\frac{\tau_{1}}{\pi(-a)^{n}} \operatorname{Re}\left[z^{n-1}\left([-i z+n y] \log \frac{z}{c}+y\right)\right] .
\end{aligned}
$$

These are the general expressions for the stress components for shear loading on one half of the boundary of the semi-plane where $n$ is restricted to integral values. As for the normal pressure cases, equations (21) include such special cases as uniform shear $(n=0)$, linearly increasing shearing forces $(n=1)$, parabolic shear $(n=2)$, hyperbolic shear where $n=-1$ and others. 
With $n$ a positive or a negative fraction, the complex function $W(z)$ becomes

$$
W(z)=\frac{\tau_{1} i}{\sin n \pi} \frac{z^{n}}{a^{n}},
$$

which when substituted in equations (11) yields the stress components

$$
\begin{aligned}
\sigma_{x} & =\frac{\tau_{1} r^{n}}{a^{n} \sin n \pi}[(n+2) \sin \phi \cdot \sin (n-1) \phi-2 \cos \phi \cdot \cos (n-1) \phi], \\
\sigma_{y} & =\frac{\tau_{1} r^{n}}{a^{n} \sin n \pi}[-n \sin \phi \cdot \sin (n-1) \phi], \\
\tau_{x y} & =\frac{\tau_{1} r^{n}}{a^{n} \sin n \pi}[\cos \phi \cdot \sin (n-1) \phi+(n+1) \sin \phi \cdot \cos (n-1) \phi] .
\end{aligned}
$$

Such special cases as parabolic shear where $n=1 / 2$, parabolic shear with $n=5 / 16$, hyperbolic shear where $n=-1 / 2$, and various others are contained in these expressions.

\section{ConClusion}

In this paper the potential method for determining stresses in a body loaded by forces in its plane was developed further than heretofore and in a somewhat different manner. The relations between the general stress components in terms of potentials for plane problems and those for the special cases of normal and shear loading on the boundary of the semi-plane were brought out. The expressions for the stress components in both the general and special cases were developed in complex form, and the displacement components were determined for both the general case and the special cases of normal and shear loading on the boundary of the semi-plane. The application of the method to various simple cases was discussed, and solutions were given for a number of more important examples of both shear and normal loading on one side of the straight semi-plane boundary.

\section{Acknowledgment}

The author desires to express his appreciation to Dr. A. Nádai of the Westinghouse Research Laboratories for having suggested the problem of developing further the potential method of stress analysis, and for his valuable help throughout the progress of the work; and to Dr. J. S. Taylor of the mathematics department of the University of Pittsburgh for his interest and support in the investigation. Acknowledgment is also due the Westinghouse Research Laboratores.

Massachusetts Institute of Technology, Cambridge, Mass. 